\title{
Integrated modelling of the edge plasma and plasma facing components
}

\author{
Coster, D.P. ${ }^{\mathrm{a}, *}$, Bonnin, X. ${ }^{\mathrm{b}}$, Mutzke, A. ${ }^{\mathrm{c}}$ Schneider, R. ${ }^{\mathrm{c}}$ Warrier, M. ${ }^{\mathrm{d}}$ \\ a Max-Planck-Institut für Plasmaphysik, EURATOM Association, Garching, Germany \\ ${ }^{\mathrm{b}}$ CNRS-LIMHP, Université Paris 13, Villetaneuse, France \\ ${ }^{\mathrm{c}}$ Max-Planck-Institut für Plasmaphysik, EURATOM Association, Greifswald, Germany \\ ${ }^{\mathrm{d}}$ Institute for Plasma Research, BHAT, Gandhinagar, Gujarat, India
}

\begin{abstract}
Modelling of the interaction between the edge plasma and plasma facing components (PFCs) has tended to place more emphasis on either the plasma or the PFCs. Either the PFCs do not change with time and the plasma evolution is studied, or the plasma is assumed to remain static and the detailed interaction of the plasma and the PFCs are examined, with no back-reaction on the plasma taken into consideration. Recent changes to the edge simulation code, SOLPS, now allow for changes in both the plasma and the PFCs to be considered. This has been done by augmenting the code to track the time-development of the properties of plasma facing components (PFCs). Results of standard mixed materials scenarios (base and redeposited $\mathrm{C} ; \mathrm{Be})$ are presented.
\end{abstract}

Key words: Edge Modelling, B2-EIRENE, SOLPS, Mixed Materials

JNM keywords: $\mathrm{P} 0500, \mathrm{~B} 0100, \mathrm{C} 0100$

PSI-17 keywords: B2/EIRENE, Edge Modelling, Divertor Modelling, Erosion \& Deposition, Mixed

Materials

PACS: 52.65.Kj, 52.25.Fi, 52.40.Hf

\section{Introduction}

The B2 component of the SOLPS package of codes[1](and references therein) has been recently extended[2-4] to include: a treatment for thermal fluxes in the wall components; an improved treatment of chemical and other sputtering processes; and the ability to model mixedmaterials.

This work describes the method used for the mixed-materials modelling, as well as some results of applying the model to ITER, where a Be

\footnotetext{
* Corresponding author: D. P. Coster, MaxPlanck-Institut für Plasmaphysik, Boltzmannstr 2, Garching bei Muenchen, D85748 Germany; David.Coster at ipp.mpg.de
}

wall and C targets are modelled.

\section{Mixed-material surface physics}

As described in [4], deposited material is tracked by the code, and a 0D time-dependent problem is solved at each position where the plasma interacts with a surface. This layer thickness is tracked, together with its composition (fraction of Be, C, etc.). For each deposited species, $i$, the number of mono-layers, $l_{i}$, is calculated. Then the fraction of deposited material exposed for sputtering is $f_{i}=\frac{l_{i}^{\beta}}{\alpha+\sum_{i} l_{i}^{\beta}}$. The contribution from the base material is then $f_{0}=1-\sum_{i} f_{i}$. Figure 1 shows the case where $\alpha=1$ (reflecting how quickly the base material disappears 


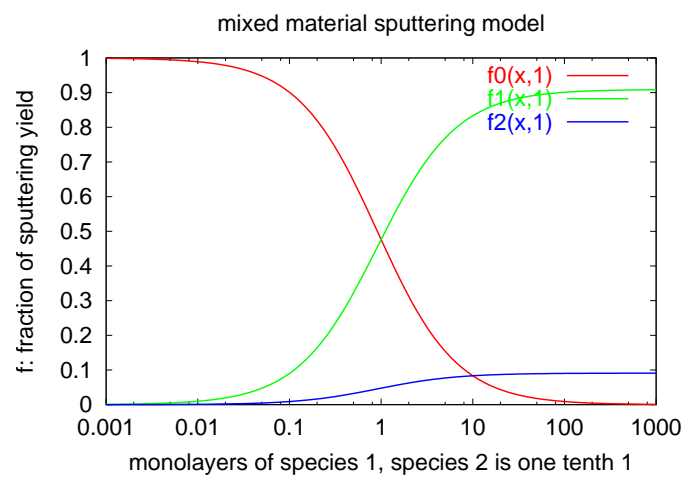

Fig. 1. The model for the fractional sputtering yield from mixed materials. $f 0$ is the sputter fraction of the base material, and $f 1$ and $f 2$ are the sputter fraction of deposited species "1" and "2", where "2" is assumed to constitute one tenth the mono-layers of " 1 ".

from the calculation) and $\beta=1$ (reflecting how quickly deposited material hides the base material). This is then used to determine the fraction of sputtered material arising from the layer (Be, $\mathrm{C}$, etc.) and from the base material. At the moment this model multiplies the rate from the basic sputtering processes (ignoring the presence of the mixed materials) by a factor giving the fractional presence of the individual materials in the mix.

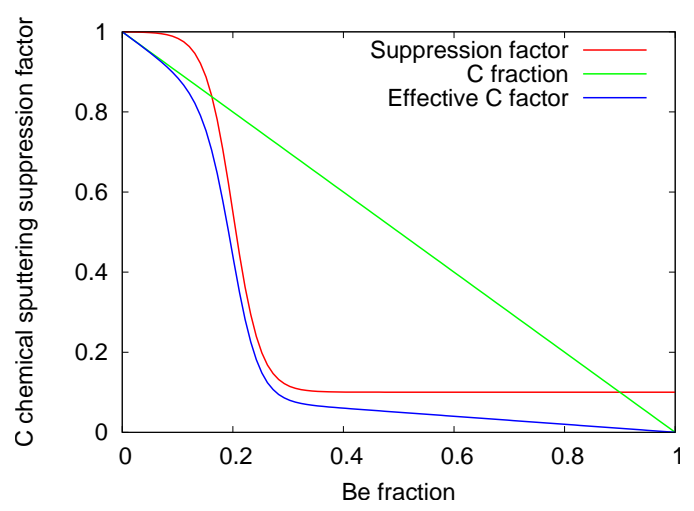

Fig. 2. Suppression factor used to lower the chemical sputtering of $\mathrm{C}$ as a function of the Be fraction. The "effective C factor" would be used to multiply the chemical sputtering coefficient. (Here it has been assumed that only Be and $\mathrm{C}$ are present.)

This has been further augmented by allowing for an enhancement factor for the chemical erosion of deposited C, and/or for a suppression of chemical erosion dependent on the local concentration of $\mathrm{Be}[5]$. The $a d$ hoc form for the suppression fac- tor is

$f_{B e}(x, a, b, c)=1-\frac{c}{2}\left(\tanh \left(\frac{x-a}{b}\right)-\tanh \left(\frac{-a}{b}\right)\right)$

with $x$ the fraction of Be, $a=0.2, b=0.05$ and $c=0.9$. The form was chosen to give a maximum suppression of $90 \%$ with a transition at about $20 \%$ Be fraction, figure 2 . This suppression factor would be multiplied by the $\mathrm{C}$ fraction and the chemical sputter yield.

\section{Results}

The simplest variant is to use only one species of $\mathrm{C}$, but to track the deposited $\mathrm{C}$ and allow it to be eroded. This provides a strong test of the coding since - if the deposited $\mathrm{C}$ is assumed to erode like the original $\mathrm{C}$ - then the plasma result should be unchanged. This has been verified, and is described in [4].

The ITER design currently foresees a mix of 3 materials to be used: $\mathrm{C}$ targets, $\mathrm{W}$ baffles and $\mathrm{Be}$ walls. At the moment, modelling with SOLPS of $\mathrm{W}$ is problematic (too many charge states and the forthcoming development of a bundled charge state model). However, some of the effects of this material mix can be simulated by limiting the calculations to $\mathrm{Be}$ and $\mathrm{C}$. We consider the case of Be walls and a $\mathrm{C}$ target.

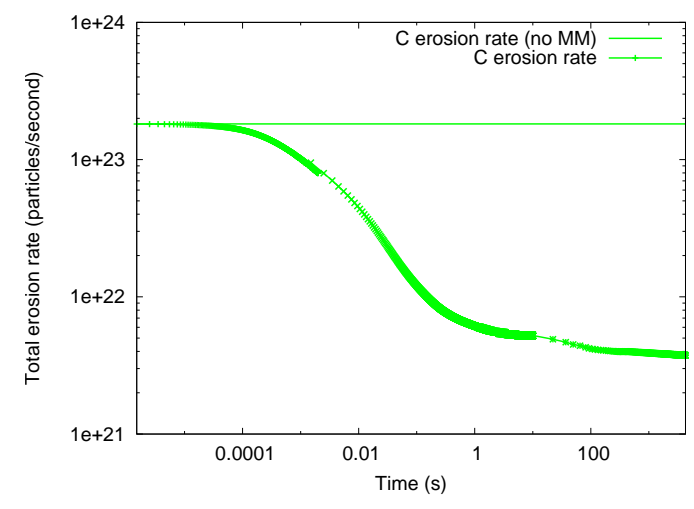

Fig. 3. Integral net $\mathrm{C}$ erosion rate, for the model without mixed materials and with mixed materials. The curve for the mixed materials combines runs with different time-steps for the plate. The integral net deposition is within $1 \%$ of the integral net erosion.

The ITER simulation used an input power crossing the inner core boundary of $100 \mathrm{MW}$, and the 

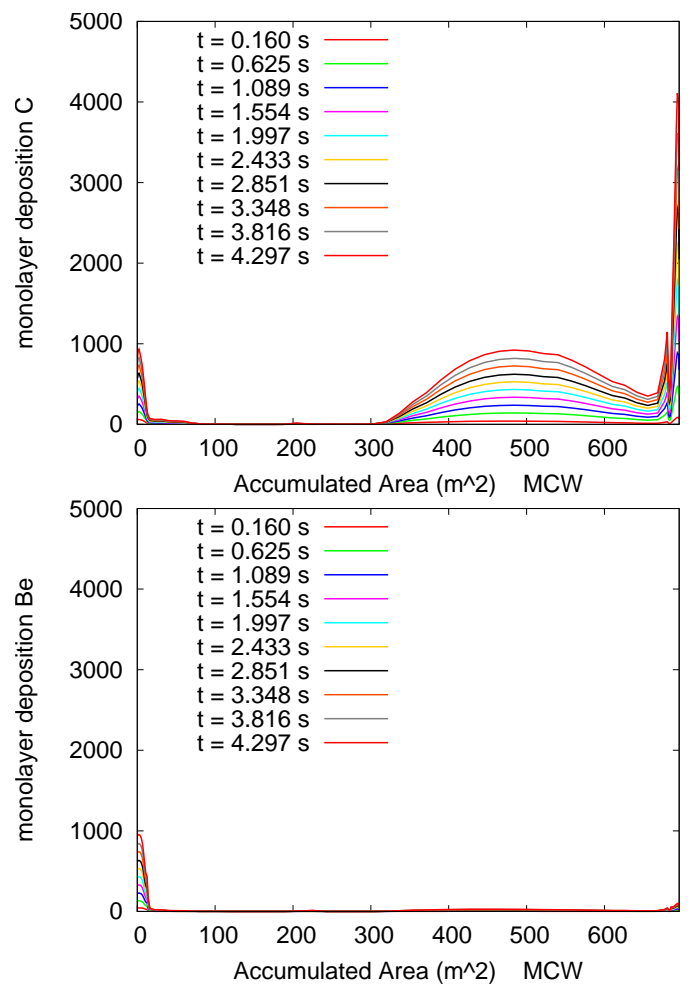

Fig. 4. Monolayers of deposition of C (top) and Be (bottom) for the main chamber wall for the ITER simulation. The $\mathrm{x}$-coordinate starts at the main chamber wall (MCW) just above the inner target and moves around the main chamber to the outer target.

density was determined by the competition between core fueling, a constant gas puff and neutral pumping through the private flux region. $\mathrm{C}$ and $\mathrm{Be}$ were produced by physical sputtering, and $\mathrm{C}$ also by chemical sputtering (with a constant yield of $2 \%$ ). The resultant simulation had a peak power flux at the outer target of just under $10 \mathrm{MWm}^{-2}$, and an upstream separatrix density of $4 \times 10^{19} \mathrm{~m}^{-3}$. Figure 3 shows the $\mathrm{C}$ erosion rate integrated over the whole surface (divertor and walls). Running without the mixed material model, $\mathrm{C}$ had a gross erosion rate of $1.8 \times 10^{23} \mathrm{~s}^{-1}$. With the mixed material model switched on the net erosion rate started at the same value, but dropped with time to end at $3.7 \times 10^{21} \mathrm{~s}^{-1}$. (Even at this time, after nearly 72 minutes, the integral net erosion rate (equal to the integral net deposition rate) is still changing.) If a $\mathrm{T}$ trapped fraction of $10 \%$ is assumed, this corresponds to around 4 1000-second ITER pulses using the initial erosion rate, and 200 1000-second pulses for the final rate (based on a $\mathrm{T}$ safety limit of $350 \mathrm{~g}$ ). These numbers are somehat crude estimates given that $3 \mathrm{~d}$ effects
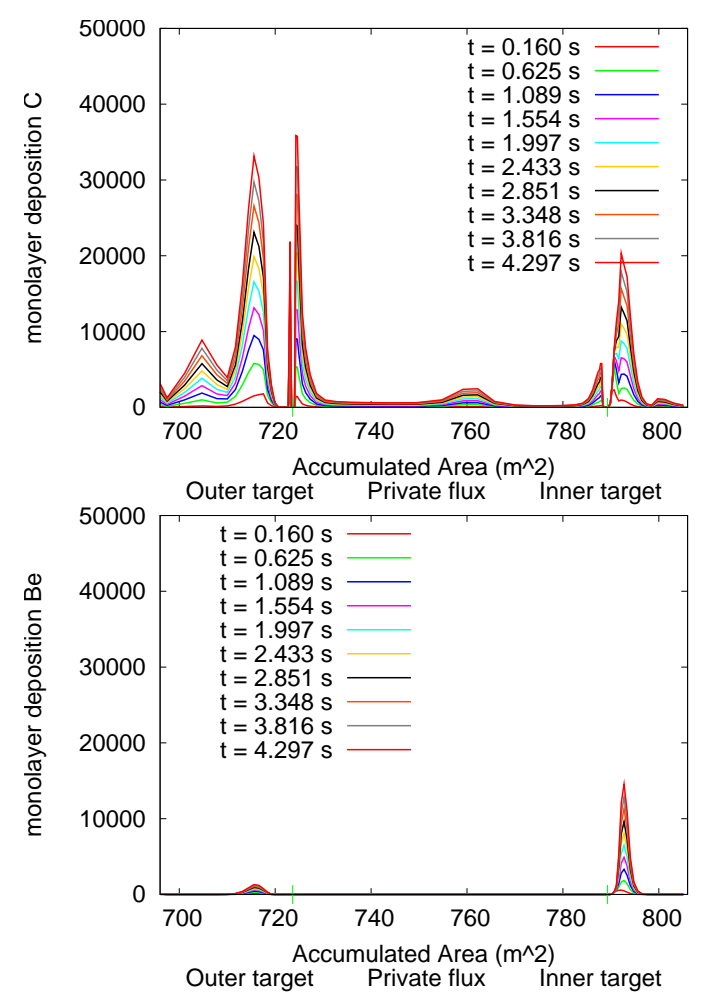

Fig. 5. Monolayers of deposition of C (top) and Be (bottom) for targets (bottom) for the ITER simulation. The $\mathrm{x}$-coordinate starts at the main chamber wall just above the outer target and moves around the divertor region to the top of the inner target. The outer and inner target strike points are at 723.6 and 789.3, respectively.

are ignored, and the assumptions that have gone into the calculation.

Figures 4 and 5 shows the deposition pattern for $\mathrm{C}$ and $\mathrm{Be}$ for the ITER simulation at the main chamber wall and at the targets. Somewhat more $\mathrm{C}$ is deposited at the outer midplane (at around $x=500 \mathrm{~m}^{2}$ ) than Be (giving a fractional $\mathrm{C}$ concentration of around $95 \%$ in the deposited material). Not much Be seems to be deposited at the outer target, but Be seems to contribute quite strongly at the inner target, at levels about half that of $\mathrm{C}$. The peak Be concentration in the plasma is about $2.5 \%$, but is diluted by $\mathrm{D}$ recycling at the inner target to about $0.03 \%$ and to less than $0.01 \%$ at the outer target.

With these fractions of $\mathrm{C}$ and $\mathrm{Be}$, suppression of $\mathrm{C}$ chemical erosion due to Be co-deposition could be expected to play a role. Calculations including these effects are underway. 


\section{Summary and Outlook}

The SOLPS edge plasma simulation package has been augmented by a model to track the erosion and subsequent deposition and re-erosion of wall and target materials. A model has been introduced to capture the essential behaviour of the resultant mixed-materials.

For a simulation of ITER, the initial large erosion rate (corresponding to gross erosion) is observed to drop significantly (by nearly a factor of 50) as re-erosion of deposited material plays an increasing role. This occurs on time-scales of more than an hour of plasma time. Somewhat unexpectedly, the simulation indicates that a $\mathrm{C}$ layer could build up on the low field side main chamber wall - in this region the deposited material is approximately $95 \% \mathrm{C}$. At the outer target, the $\mathrm{C}$ fraction is in excess of $95 \%$, whereas in parts of the inner divertor, Be fractions of $40 \%$ are found.

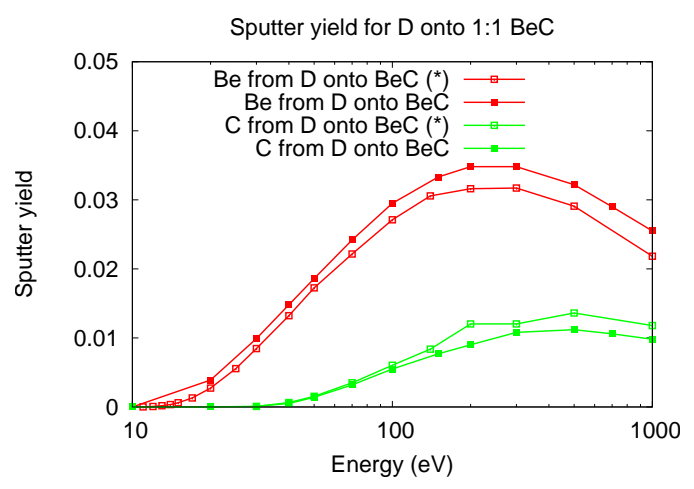

Fig. 6. Physical sputtering yields of Be and $\mathrm{C}$ produced by $\mathrm{D}$ bombardment of a 1:1 mixture of $\mathrm{Be}$ and $\mathrm{C}$. The lines labeled with $\left(^{*}\right)$ indicates that the single species TRIM data[6] were used, and scaled by the relative fraction of the $\mathrm{Be}$ or $\mathrm{C}$ in the mixture ( $\frac{1}{2}$ in this case).

It is planned to improve the somewhat ad hoc mixed-materials sputtering models used by use of $3 \mathrm{~d}$ data sets based on TRIM calculations (angle, incoming particle energy, fraction of (say) $\mathrm{C}$ in Be/C layer). For lower energies (where chemistry can be expected to play a larger role), these data sets should be enhanced by molecular dynamics calculations or specific low energy experiments. As an example, the physical sputtering yield from D impinging on a 50-50 mixture of $\mathrm{BeC}$ is shown in figure 6 . For this case, the differ- ence between the assumption used in this work and the results of TRIM are small. For cases involving $\mathrm{W}$, the differences can be larger, with the yields of the lighter species being considerably under-estimated with the simple model. In addition to the sputtering rates, surface and bulk properties of Be-C and Be-C-W such as melting temperatures, vapour pressures, emissivities, heat capacities and thermal conductivities are also needed.

In the near future, the ADAS project[7] is planning to release a bundled charge model for $\mathrm{W}$, and this means that it should soon be possible to extend the C-Be calculations to C-W, Be-W and to $\mathrm{C}-\mathrm{Be}-\mathrm{W}$.

The mixed-materials modifications should also be included in the Eirene part of SOLPS as well.

\section{References}

[1] R. Schneider, X. Bonnin, K. Borrass, D. P. Coster, H. Kastelewicz, et al., Contrib. Plasma Phys. 46 (2006) 3, DOI 10.1002/ctpp.200610001.

[2] D. P. Coster, X. Bonnin, A. Chankin, G. Corrigan, S. K. Erents, et al., in Proc. of the 20th IAEA Conference Fusion Energy (CD-Rom), Vilamoura, Portugal, November 2004, volume IAEA-CSP-25/CD, pages IAEACN-116/TH/P5-18, Vienna, 2005, IAEA.

[3] M. Warrier, X. Bonnin, R. Schneider, and D. Coster, in Europhysics Conference Abstracts (CD-ROM, Proc. of the 30th EPS Conference on Controlled Fusion and Plasma Physics, St. Petersburg, 2003), volume 27B, Geneva, 2003, EPS.

[4] D. Coster, X. Bonnin, and M. Warrier, Physica Scripta T124 (2006) 9.

[5] R. Doerner, M. Baldwin, and K. Schmid, Physica Scripta T111 (2004) 75.

[6] W. Eckstein, IPP-Report 9/117, Max Planck Institute for Plasma Physics, Garching, 1998.

[7] Atomic Data and Analysis Structure: http://adas.phys.strath.ac.uk/. 